green, and violet - I will not enter into here, but merely remark that I found, as other observers have, the first much the more frequent, whilst of the last only three cases occurred, and these by no means so marked as the other forms. As to the value of these results, I admit that they of course depend on the exactitude of my observations. I therefore would say that from familiarity with the test I feel perfectly sure that I detected color-blindness where it existed. I always pushed the test much further than would seem necessary, often thereby causing remark and question from the bystanders. Although seemingly tedious and monotonous from the tax upon the eye and ear and voice of the examiner, I found my tests of great psychological interest, frequently shared in by educated observers.

Since this paper was read at the society I have received the reports of Drs. Hermann Cohn and Hugo Magnus in Breslau, who the past winter have been like myself engaged in the examination of schoolchildren for color-blindness. They employed the same method as myself, namely, Professor Holmgren's, and their results agree with mine above given. Among 2761 boys they found 76, or 2.7 per cent., colorblind. Among 2318 girls they found only one color-blind, or 0.04 . This defect seems, therefore, a very great rarity among females. Color-blindness occurred twice as often among Jewish children as among Christians. The single case among girls which Dr. Cohn found was one of red-green and blue-yellow blindness, quite atypical.

\title{
TWO CASES OF EMPYEMA; RAPID RECOVERY AFTER OPERATION.
}

BY W. H. WORKMAN, M. D., WORCESTER.

Case I. A. B., a boy of five years, came under the notice of Dr. Henry Clarke upon the 14th of April, 1875, having been ill about three weeks. He was much emaciated, very feeble, and was bathed in perspiration. His temperature was considerably elevated. He had been allowed but little nourishment, and was said to have been treated for " disease of the liver." Examination revealed extensive effusion in left side of chest. The next day, April 15th, the aspirator was used, and three pints of pus were withdrawn. After the operation the child improved, and took nourishment and stimulants freely. April 25th, the chest having refilled, Dr. Clarke made a permanent opening in the eighth intercostal space below the angle of the scapula. A considerable quantity of very offensive pus was evacuated. The cavity was thoroughly washed out with a weak solution of carbolic acid. Stimulants, liquid nourishment, and tinct. ferri chloridi were prescribed, and the pleural cavity was to be washed out daily with a solution of carbolic 
acid. This treatment was carried out chiefly under the supervision of the writer, and the further progress of the case was as follows :-

On the day after the operation all fætor had vanished. There was but little discharge, and that sero-purulent. The child was comfortable and much brighter. From this time the discharge diminished daily in quantity, was chiefly serous, and was destitute of odor. The strength improved, and cough was not urgent. The lung, which had been much compressed, expanded and resumed its natural position. About five weeks after the permanent opening was made, respiration could be heard quite to the base of the chest, of good quality, and unattended by râles or friction sounds. The diseased side was somewhat flattened. The opening was now allowed to close, and all symptoms being favorable the patient was discharged. A few months ago he was seen and examined by the writer, who could discover no deformity, nor other trace of disease. The left side was in every respect the equal of the right side of the chest.

CASE II. On the evening of August 10, 1877, the writer was called to a child three years of age, who had been ill for a month with cough accompanied with fever and thirst. When seen the patient was lying with half-closed eyes, apparently unmindful of what was taking place around him. He was pale, emaciated to a skeleton, and coughed frequently. Respiration was moaning, and about fifty per minute. Temperature in axilla $99.2^{\circ} \mathrm{F}$; p pulse 160 . The left side of the chest bulged considerably, the intercostal furrows being obliterated, while upon the right side they were strongly marked. Percussion of the left side revealed greatly diminished resonance above the second rib in front and in the supra-scapular fossa ; thence absolute flatness to the base both in front and behind. Upon auscultation distant respiration without râles or friction sounds could be heard over the whole of the left side, evidently transmitted from the right side, since the quality of the respiration on the two sides was the same. The respiratory murmur of the right side was harsh, but attended with no râles. The heart was pulsating almost entirely to the right of the sternum, the apex impulse and systolic sound being situated about an inch to the right of this bone in the fifth intercostal space.

Stimulants were freely given during the night, and the next morning, with the assistance of Dr. J. B. Rich, I withdrew with the aspirator forty-six ounces of odorless, chocolate-colored pus, the puncture being made in the eighth intercostal space, a little outward from the angle of the scapula. Reversing the action of the instrument, the pleural cavity was thoroughly washed out with a one per cent. solution of carbolic acid. No severe coughing followed the operation. Immediately afterwards the child fell asleep, and the pulse, which previous to evacuation of the fluid was 166 , fell to 148 . 
The next morning the patient was much brighter, noticed all that was being done in the room, and called for and was allowed to eat a small " fried pout." Temperature under the tongue $99^{\circ}$; pulse 144 .

The after-treatment was conducted as follows: (1.) The patient was well protected with flannel. (2.) Concentrated liquid nourishment was given hourly in such quantities as could be well borne. (3.) Opium was taken sufficiently often to allay undue irritability. (4.) Tinct. ferri chloridi was administered in whisky every six hours, and lager beer was allowed ad libitum.

During the eight days following the operation the pulse ranged from 136 to 140 , and the temperature from $98^{\circ}$ to $99^{\circ}$. Upon the ninth and tenth days, after exposure to cold at night, the temperature rose to $100.6^{\circ}$ and $101.4^{\circ}$ respectively. It then fell to the normal point, above which it did not again rise. Appetite and strength steadily improved. Cough was at no time marked.

September 8th, twenty-eight days after aspiration of chest, the condition of the patient was the following: color good ; respiration easy and regular; bowels open; appetite good; no cough; tongue clean; pulse 100 ; temperature $98.4^{\circ}$.

The left side was somewhat flattened and not so free in its movements as the right. The percussion sound was a little tympanitic. The respiration and vocal resonance were fainter than on the right side, but of good quality, and heard to the base of the chest. There were no râles or friction sounds. The heart beat in the normal position.

At date of writing, February 16,1878 , the child is in the enjoyment of perfect health, and exhibits no sign of his former disease.

These cases illustrate the success which may attend the surgical treatment of empyema, even when the circumstances before operation are such as to render the prognosis unfavorable or very doubtful. Both of these patients were in an extremely wretched condition, so that no opinion as to prognosis could be ventured at the time of the aspiration of the chest. In Case II. the operation was urged as giving only a chance of saving the life of the child, who was apparently moribund.

Noteworthy are the complete change within twenty-four hours in the character of the discharge and its subsequent scantiness in Case I.; the fact that the employment of aspiration alone, and that only once, was necessary in Case II.; and the rapid and complete recovery made in both. 